\title{
Monoenergetic laser wakefield acceleration
}

\author{
N. E. Andreev and S. V. Kuznetsov \\ High Energy Density Research Center, IVTAN, Moscow, 127412, Russia
}

I. V. Pogorelsky

Accelerator Test Facility, BNL, Upton, New York 11973

(Received 15 January 1999; revised manuscript received 10 September 1999; published 17 February 2000)

\begin{abstract}
Three dimensional test particle simulations are applied to optimization of the plasma-channeled laser wakefield accelerator (LWFA) operating in a weakly nonlinear regime. Electron beam energy spread, emittance, and luminosity depend upon the proportion of the electron bunch size to the plasma wavelength. This proportion tends to improve with the laser wavelength increase. We simulate a prospective two-stage $\sim 1 \mathrm{GeV}$ LWFA with controlled energy spread and emittance. The input parameters correspond to realistic capabilities of the BNL Accelerator Test Facility that features a picosecond-terawatt $\mathrm{CO}_{2}$ laser and a high-brightness electron gun.
\end{abstract}

PACS numbers: 29.17.+w, 41.75.-i, 52.35.-g, 29.27.Fh

\section{INTRODUCTION}

Successful experimental demonstration of ultrahigh gradient (up to $100 \mathrm{GeV} / \mathrm{m}$ ) laser acceleration of electrons in plasma [1-4] gave an impetus to further development of new particle accelerator concepts which are alternatives to the conventional rf linac. The advancement of laser accelerators toward practically meaningful devices is foreseen through realization of quasimonoenergetic acceleration over extended distances and for appreciably bulk electron charges.

A predicament for producing highly monoenergetic, low emittance electron beams is the formation of small size bunches to compare with the wavelength of the accelerating oscillatory field. The frequency of plasma wakefield in laser accelerators, typically driven by femtosecond solid state lasers, is orders of magnitude higher as compared with conventional rf linacs. This requires proportionally short electron bunches. Existing electron injection devices, such as photocathode rf guns, do not fit these schemes. Therefore, novel laser methods to trap ultrashort electron bunches in the plasma wave have been proposed [5,6], and their feasibility is the subject of further analysis.

In this paper, we approach the monoenergetic laser acceleration from another position. The proposed concept is based on using conventional high-brightness electron injectors in combination with the emerging picosecondterawatt (ps-TW) $\mathrm{CO}_{2}$ lasers. The first ps-TW $\mathrm{CO}_{2}$ laser is being commissioned at the BNL Accelerator Test Facility [7-9]. Because of 10 times longer wavelength $\lambda$, to compare with the $T^{3}$ solid state lasers, the $\mathrm{CO}_{2}$ laser opens new opportunities for LWFA development that we explore in this paper.

Because of the superior stability and regularity of the plasma wake, the laser wakefield accelerator (LWFA) [10-12] operating in the linear regime is generally considered as the preferable scheme. In the standard LWFA scheme, the plasma wave is excited by a short laser pulse close in duration to the half-period of the plasma, $\tau_{L} \approx$ $\lambda_{p} / 2 c$, that depends upon the ambient electron plasma density $n_{e}: \lambda_{p}[\mu \mathrm{m}]=3.3 \times 10^{10} n_{e}^{-1 / 2}\left[\mathrm{~cm}^{-3}\right]$. The maximum amplitude of the accelerating plasma wakefield is $[12,13]$

$$
E_{a}^{\max }[\mathrm{GV} / \mathrm{m}]=2.8 \times 10^{4}\left(\frac{\lambda}{r_{L}}\right)^{2} P_{L}[\mathrm{TW}] / \lambda_{p}[\mu \mathrm{m}],
$$

where $P_{L}$ is the laser power and $r_{L}$ is the radius of the laser focus spot. Thus, the increase in the plasma density (i.e., decrease of $\lambda_{p}$ ) leads to higher accelerating field.

Another important accelerator parameter is the maximum number of electrons per bunch, which is defined by the condition that the self-field of the bunch does not affect the plasma wakefield structure,

$$
N_{e} \ll n_{e}\left(c / \omega_{p}\right)^{3}=4 \times 10^{6} \lambda_{p}[\mu \mathrm{m}] .
$$

This condition favors bigger $\lambda_{p}$.

A similar conclusion can be drawn from the requirement of a small energy spread of the accelerated electrons, which can be satisfied when the electron bunch is much shorter than the plasma wake period, $\tau_{b} \ll \lambda_{p} / c$. Therefore, a certain tradeoff needs to be resolved when one wishes to design the high-gradient laser accelerator capable of producing a high-brightness electron beam.

Here we explore a possible solution to this problem dwelling on the $\lambda^{2}$-proportional enhancement of the plasma wakefield shown in Eq. (1). Because of the enhanced ponderomotive action of the long-wavelength radiation, the $\mathrm{CO}_{2}$ laser $(\lambda=10 \mu \mathrm{m})$, used instead of a solid state laser $(\lambda=1 \mu \mathrm{m})$, allows the reduction of the plasma density in the LWFA without jeopardizing the net acceleration. Then, a conventional photocathode electron gun appears to be a suitable injector for the LWFA with controlled beam properties. 
In Sec. II, we describe the 3D (axisymmetric) test particle simulations of the $1.5 \mathrm{GeV}$ LWFA driven by the prospective $50 \mathrm{TW}, 1 \mathrm{ps} \mathrm{CO}_{2}$ laser with the $50 \mathrm{MeV}$ linac used as an injector. These results show that even with a relatively short electron bunch, $L_{b}=0.1 \lambda_{p}$, the energy spread of the accelerated electrons may exceed $50 \%$.

To minimize the energy spread, in Sec. III we analyze the conditions of the electron bunch compression by the laser wakefield. Then, we apply our findings to improve the energy spread in the LWFA. As shown in Sec. IV, the $1.5 \mathrm{GeV}$ plasma-channeled LWFA operating in a weakly nonlinear regime can produce the electron beam with $<2 \%$ energy spread when the LWFA buncher is used between the conventional $5 \mathrm{MeV}$ electron injector and the LWFA acceleration stage. The possibility of multi-GeV quasimonoenergetic acceleration using multistage LWFA is also demonstrated.

\section{DEPENDENCE OF THE LWFA PERFORMANCE UPON THE ELECTRON BUNCH DURATION}

To study the acceleration of relativistic electrons in the wakefield we use the equations of motion in the form [14]:

$$
\begin{gathered}
\frac{d P_{z}}{d \tau}=F_{z}(\xi, \rho), \\
\frac{d \boldsymbol{P}_{r}}{d \tau}=\boldsymbol{F}_{r}(\xi, \rho), \\
\frac{d \xi}{d \tau}=\frac{P_{z}}{\sqrt{1+P_{z}^{2}+P_{r}^{2}}}-\beta, \\
\frac{d \rho}{d \tau}=\frac{\boldsymbol{P}_{r}}{\sqrt{1+P_{z}^{2}+P_{r}^{2}}},
\end{gathered}
$$

where $P_{z}, \boldsymbol{P}_{r}=\left\{P_{x}, P_{y}\right\}$ are the longitudinal and radial components of the electron momentum normalized to $\quad m c, \quad \xi=k_{p}\left(z-V_{\mathrm{ph}} t\right), \quad \rho=k_{p} \boldsymbol{r}=k_{p}\{x, y\}=$ $k_{p} r\{\cos \varphi, \sin \varphi\}, \quad \varphi=\arctan (y / x)$ are the dimensionless coordinates of the electron, $\tau=\omega_{p} t$, where $\omega_{p}=\sqrt{4 \pi e^{2} n_{e} / m}$ is the electron plasma frequency, $k_{p}=\omega_{p} / c$ is the wave number of the plasma wave, and $\beta=V_{\mathrm{ph}} / c$ is the dimensionless phase velocity of the wake. The axial and radial components of the normalized force acting on the electron moving with the velocity $c$ along the $0 Z$ axis can be expressed in terms of the normalized (to $m c^{2} / e$ ) wakefield potential $\phi$ as follows $\left(\boldsymbol{F}_{r}=\left\{F_{x}, F_{y}\right\}=F_{r}\{\cos \varphi, \sin \varphi\}\right):$

$$
\begin{gathered}
F_{z} \equiv \frac{e E_{z}}{m c \omega_{p}}=\frac{\partial \phi}{\partial \xi}, \\
F_{r} \equiv \frac{e E_{r}}{m c \omega_{p}}-\frac{e B_{\varphi}}{m c \omega_{p}}=\frac{\partial \phi}{\partial \rho} .
\end{gathered}
$$

To define a laser wakefield potential we use a set of relativistic hydrodynamic equations for cold background plasma electrons and Maxwell's equations. We consider the axisymmetrical geometry where immobile background plasma density distribution $n_{e}(r)$ is centered along the axis $0 Z$ and depends only on the radial variable $r$ (preformed plasma channel), and the symmetrical laser pulse propagates along the same axis $0 Z[15,16]$. In this case, in the quasistatic approximation (valid when the laser pulse does not change significantly over the propagation length comparable with the pulse duration) [12] and assuming sufficiently rarefied plasma $\left(V_{\mathrm{ph}} \approx c\right)$, the wakefield potential depends primarily on variables $\rho$ and $\xi$.

Previous studies of the nonlinear dynamics of the laser pulse propagating in the preformed plasma channel [16] with the density parabolically rising in the radial direction

$$
n_{e}(r)=n_{e}(r=0)\left[1+\frac{r^{2}}{R_{\mathrm{ch}}^{2}}\right]
$$

demonstrate that the Gaussian laser pulse with the normalized laser vector potential

$$
a=e E_{L} / m c \omega=a_{0} \exp \left[-\frac{r^{2}}{2 r_{L}^{2}}-(2 \ln 2) \frac{\left(\xi-\xi_{0}\right)^{2}}{\left(\omega_{p} \tau_{L}\right)^{2}}\right],
$$

where $\tau_{L}$ is the pulse full width at half maximum of the intensity (FWHM) in the longitudinal direction, does not experience envelope oscillations if the laser spot radius $r_{L}$ is matched to the radius of a parabolic plasma channel Eq. (9) by the condition $R_{\mathrm{ch}} \approx k_{p} r_{L}^{2}[17,18]$ and the laser peak power does not exceed the self-focusing threshold $P_{\text {sf }} \cong 16.2 \frac{n_{\text {cr }}}{n_{e}}[\mathrm{GW}]$.

Consider the laser pulse propagation in the matched parabolic channel. To calculate the wakefield structure in the plasma channel Eq. (9) by the method described in [15] we used a set of equations [Eqs. (7)-(10)] [16]. The plasma density along the channel axis $n_{e}(0)$ is chosen by the condition for the excitation of a wakefield by the Gaussian pulse in a homogeneous plasma $\omega_{p} \tau_{L}=$ $2 \sqrt{2 \ln 2}[11,12]$.

Three dimensional test particle simulations of Eqs. (3)$(10)[19,20]$, that describe electron acceleration by plasma wake excited in the matched parabolic plasma channel, help to verify the conditions for practically achievable monoenergetic LWFA. The following input parameters have been used in simulations: $\mathrm{CO}_{2}$ laser $(\lambda=10 \mu \mathrm{m})$ with the pulse duration $\tau_{L}=1 \mathrm{ps}$ and power $P_{L}=50 \mathrm{TW}$ focused to $r_{L} \cong 500 \mu \mathrm{m}$ that defines $a_{0}^{2}=0.5$ (weakly nonlinear regime); plasma parameters $\lambda_{p}=800 \mu \mathrm{m}$, that corresponds to the plasma density $n_{e}(0)=1.6 \times 10^{15} \mathrm{~cm}^{-3}, \quad k_{p} R_{\mathrm{ch}}=14.3, \quad k_{p} r_{L}=3.8$. Note that $\lambda_{p}$ and $k_{p}$ are defined at the axis of the plasma channel. The maximum accelerating gradient calculated by Eq. (1) is $E_{a}^{\max } \cong 0.7 \mathrm{GV} / \mathrm{m}$.

In the simulations discussed in this section, we considered the $50 \mathrm{MeV}$ injected electron bunch $\left(E_{\text {inj }}=\right.$ $100 m c^{2}$ ) with the energy spread $0.2 \%$, normalized emittance $2 \mathrm{mmmrad}$, and the $250-500 \mathrm{fs}(80-160 \mu \mathrm{m})$ bunch length. 


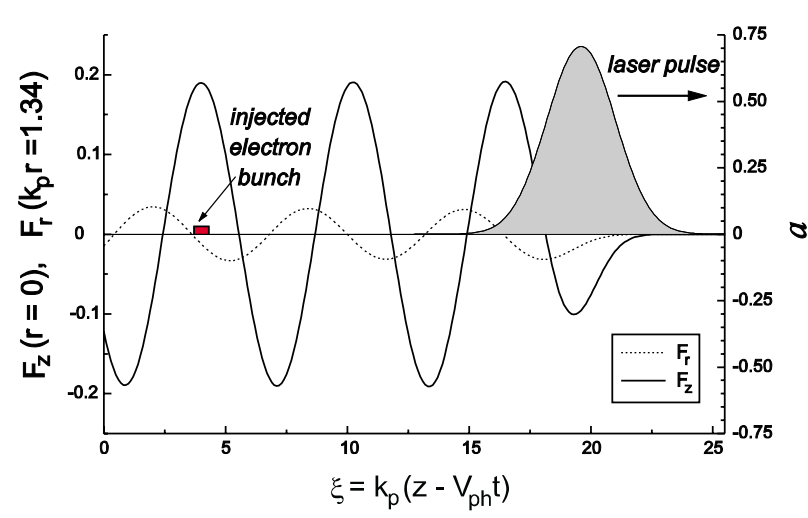

FIG. 1. (Color) Accelerating $F_{z}$ and focusing $F_{r}$ wakefield forces in a matched parabolic plasma channel; $\mathrm{CO}_{2}$ laser $\tau_{L}=$ $1 \mathrm{ps}, P_{L}=50 \mathrm{TW}, a^{2}=0.5$; plasma parameters $k_{p} r_{L}=3.8$, $k_{p} R_{\mathrm{ch}}=14.3, \lambda_{p}=800 \mu \mathrm{m}$.

As demonstrated in Fig. 1, the above plasma and laser parameters lead to excitation of fairly regular wakefield. Because of the weak nonlinearity of the excited wakefield potential and radial nonhomogeneity of the background plasma density in the channel, the beginning of the focusing phase is slightly shifted from the accelerating field maximum. This effect, which is more pronounced for a smaller channel radius and higher wakefield amplitudes, increases the overlapping of focusing and accelerating phases (in comparison with the linear wakefield in a homogeneous plasma), and, consequently, increases the accelerating length and maximum energy gain of electrons $[16,21]$. Therefore, for the electron bunch injection we choose the wakefield phase near the maximum of the accelerating field close to the beginning of the focusing phase.

The longitudinal phase space of an accelerated electron bunch along the acceleration length $L_{\text {acc }}$ for the initially homogeneous electron bunch of the length $L_{b 0} \equiv \tau_{b 0} c=$ $0.1 \lambda_{p}=80 \mu \mathrm{m}$ (or $270 \mathrm{fs}$ ) and radius $r_{b}=L_{b 0}$ is shown in Fig. 2. The maximum dephasing length, $L_{\mathrm{ph}}=$

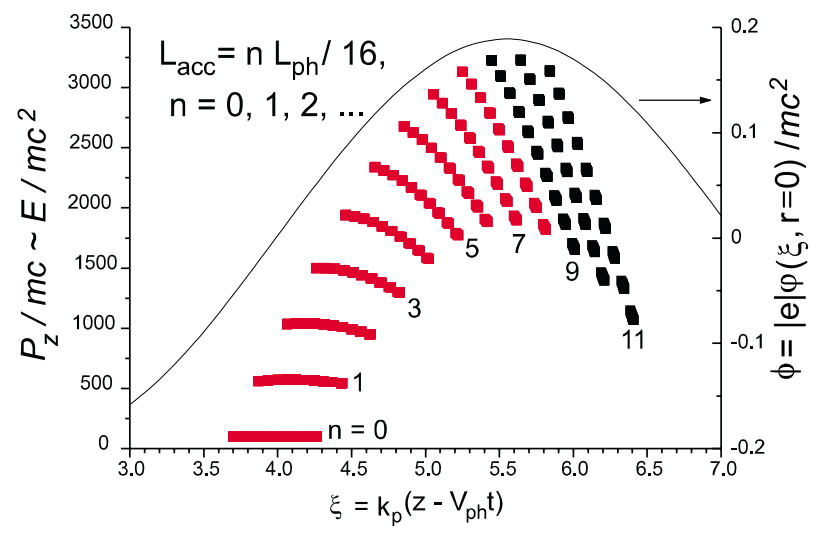

FIG. 2. (Color) Wakefield acceleration of an electron bunch by channel guided $\mathrm{CO}_{2}$ laser pulse; $a=0.71$, $k_{p} r_{L}=3.8, \quad k_{p} R_{\mathrm{ch}}=14.3, L_{\mathrm{ph}}=512 \mathrm{~cm}, E_{\mathrm{inj}}=100 m c^{2}$, $L_{b}=r_{b}=0.1 \lambda_{p}$. $\left(1-\beta^{2}\right)^{-1} \lambda_{p} \equiv \gamma^{2} \lambda_{p}=\gamma^{3} \lambda$, corresponds to the $\lambda_{p} / 2$ electron slippage relative to the wakefield. Simulations of the electron bunch acceleration in the plasma wake illustrated by Fig. 2 demonstrate more than $50 \%$ energy spread to the end of the acceleration cycle $\left(L_{\mathrm{acc}} / L_{\mathrm{ph}} \cong\right.$ $0.5)$ when up to $1.6 \mathrm{GeV}$ net energy gain is achieved. As seen from Fig. 2, the finite bunch length seems to be the prime cause for the significant energy spread obtained.
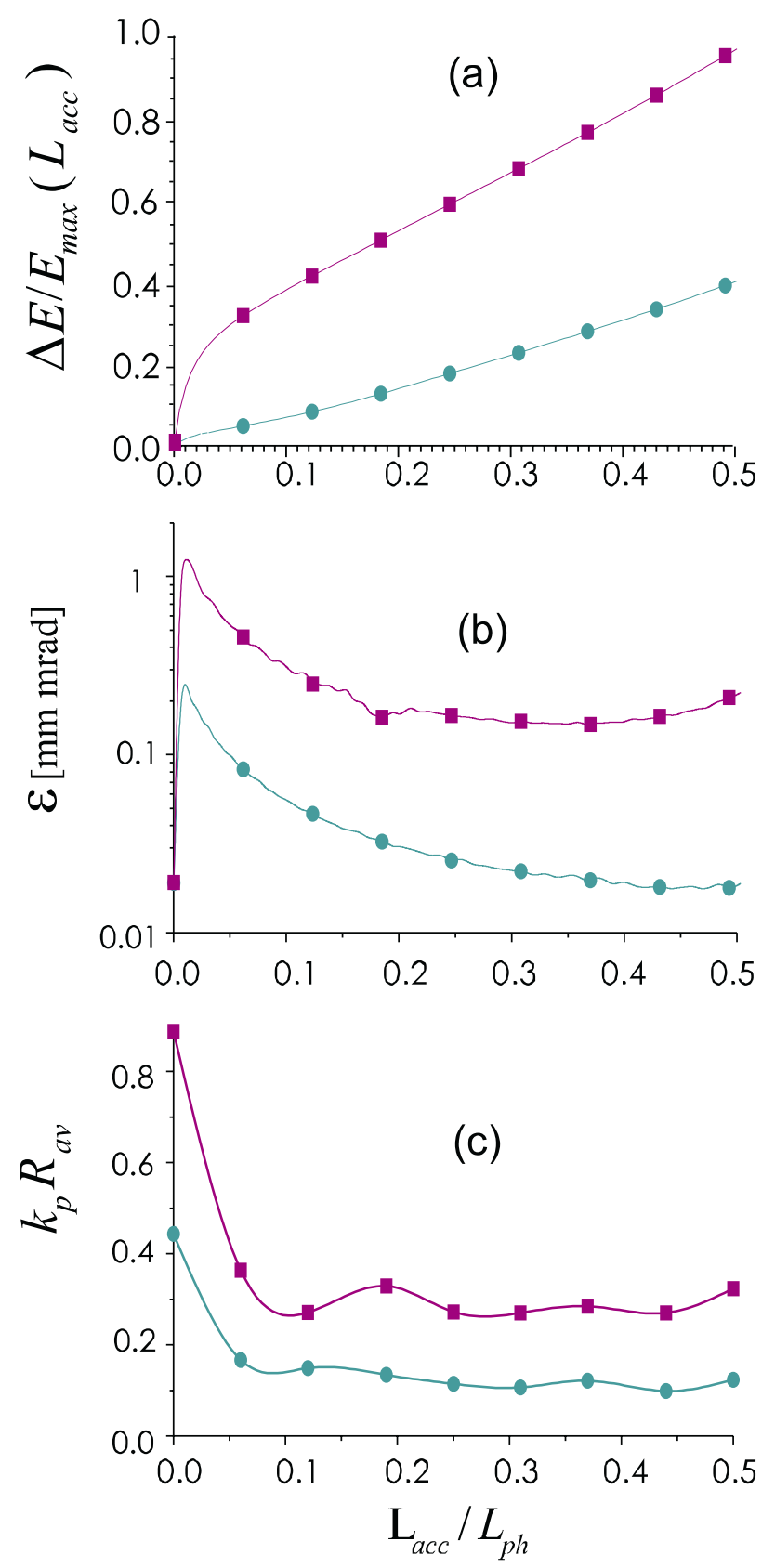

FIG. 3. (Color) Quality of an accelerated electron bunch in channel guided LWFA; $a=0.71, k_{p} r_{L}=3.8, k_{p} R_{\mathrm{ch}}=14.3$, $E_{\text {inj }}=100 m c^{2}, L_{\mathrm{ph}}=512 \mathrm{~cm}$; (a) energy spread; (b) geometric emittance; (c) average $e$-beam radius; circles: $L_{b}=r_{b}=$ $0.1 \lambda_{p}$; boxes: $L_{b}=r_{b}=0.2 \lambda_{p}$. 
Figure 3 shows how the electron beam quality depends upon the initial bunch size $L_{b 0}=r=0.1-$ $0.2 \lambda_{p}$. Simulations demonstrate the importance of using possibly small (especially in the longitudinal direction) electron bunches in order to control the beam quality in the course of acceleration. Figures 3(b) and 3(c) show that strong bunch focusing in the wakefield allows the maintenance of a low geometric emittance $\varepsilon=4\left[R_{a \nu}^{2} \Theta_{a \nu}^{2}-\langle r \theta\rangle^{2}\right]^{1 / 2}$, where $R_{a \nu}=\left\langle r^{2}\right\rangle^{1 / 2}$ is the bunch rms radius and $\Theta_{a \nu}=\left\langle\theta^{2}\right\rangle^{1 / 2}\left(\theta=P_{r} / P\right)$ defines the angular divergence of the electron beam. However, the selected initial bunch duration does not permit the achievement of a low energy spread which dramatically increases with the increase in the initial electron bunch length [see Fig. 3(a)].

\section{BUNCH COMPRESSION IN THE LASER WAKEFIELD}

In this section, we study the possibility of electron bunch longitudinal compression in the plasma wakefield in order to minimize the bunch length at the input to the accelerating stage. The physical mechanism of bunch compression is due to the longitudinal gradient of the force $F_{z}$. At the proper injection phase on the negative slope of the oscillating field, where $\partial^{2} \phi / \partial \xi^{2}<0$, the accelerating force at the front of the bunch is smaller than at the back. The strongest bunch compression will take place in the area of the highest negative gradient $\partial F_{z} / \partial \xi$, i.e., in the vicinity of the maximum wakefield potential $\phi_{\max }=\phi\left(\xi_{m}\right)$. It has been demonstrated [22] that, for the electrons below the resonance energy $\left(E_{\text {in j }}<\gamma m c^{2}\right)$ injected at phase $\xi_{m}$, sharp reduction of the bunch length takes place in the vicinity of the trapping point $\xi_{t r}$ (turning point of the electron motion relative to the plasma wave). Thus, the minimum length of the bunch is determined primarily by its initial length $L_{b 0}$ and by the wakefield potential in the points $\xi_{m}$ and $\xi_{t r}$ (not taking into account the radial motion of electrons),

$$
L_{b}=\frac{k_{p} L_{b 0}^{2}}{2} \frac{\left|\partial^{2} \phi\left(\xi_{m}\right) / \partial \xi^{2}\right|}{\partial \phi\left(\xi_{t r}\right) / \partial \xi} .
$$

This equation shows that, for effective compression, the initial length of the electron bunch should not exceed the skin depth $k_{p}^{-1}$. The compression factor $L_{b 0} / L_{b}$ increases with the reduction of the initial bunch length and does not depend directly on the amplitude and phase velocity of the wakefield, as long as the electron capture condition is satisfied. This condition appears as follows:

$$
\begin{aligned}
\phi_{\max }-\phi\left(\xi_{t r}\right)= & E_{\text {in }} / m c^{2} \\
& -\left[\left(1-\gamma^{-2}\right)\left(E_{\text {inj }}^{2} / m^{2} c^{4}-1\right)\right]^{1 / 2} \\
& -1 / \gamma .
\end{aligned}
$$

For example, for a wakefield with $\gamma=80$ and electron injection energy $E_{\mathrm{inj}}=5 \mathrm{MeV} \approx 10 m c^{2}$, the amplitude of the wakefield sufficient for the trapping of an injected bunch appears to be relatively small, $|e| \phi_{\max } / m c^{2} \geq 0.04$.

In addition, for steady compression of electron bunches, the whole area of electron longitudinal motion $\xi \in$ $\left[\xi_{t r}, \xi_{m}\right]$ should be within the focusing phase of the wakefield. Hence, just half of the dephasing length can be used for acceleration.

Figure 4 illustrates the results of simulations of the electron bunch compression in the wakefield with $\gamma=80$ and amplitudes $\phi_{\max }=0.04-0.19$ that correspond to a weakly nonlinear regime. The initial parameters of the electron bunch at the entrance to the plasma channel are $E_{\text {inj }}=5 \mathrm{MeV}$, energy spread $1.5 \%$, geometric emittance $0.6 \mathrm{~mm} \mathrm{mrad}$, length $L_{b 0}=30 \mu \mathrm{m}(100 \mathrm{fs})$, and $\mathrm{rms}$ radius $R_{a \nu}=25-50 \mu \mathrm{m}$. The laser spot size and plasma parameters are taken the same as in the simulations discussed in Sec. II.

It is seen from Fig. 4(a) that a significant (more than 5 times) bunch compression can be attained for different wakefield amplitudes, in accordance with Eq. (11).
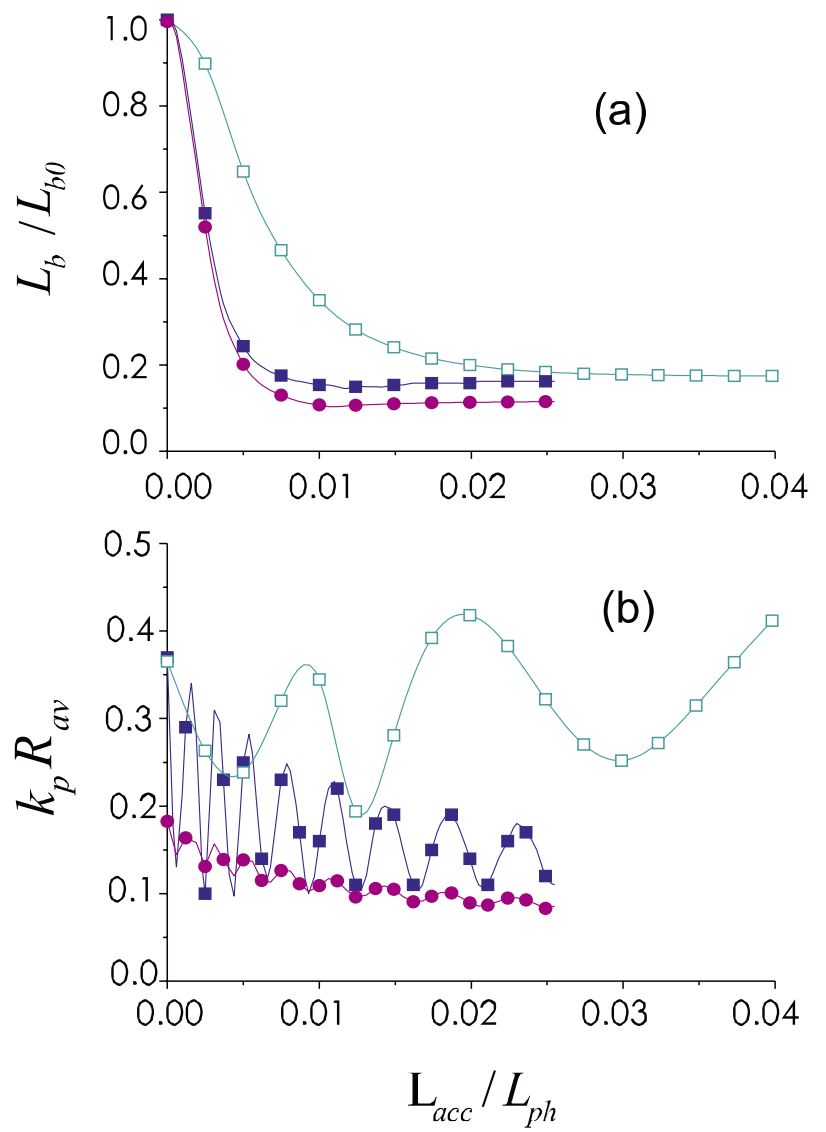

FIG. 4. (Color) Bunch compression in channel guided LWFA; plasma channel parameters: $\lambda_{p}=800 \mu \mathrm{m}, k_{p} r_{L}=3.8$, $k_{p} R_{\mathrm{ch}}=14.3$; initial bunch energy $E_{\mathrm{inj}}=5 \mathrm{MeV}$, energy spread $1.5 \%$, normalized emittance $\left(\varepsilon_{n}=0.6 \mathrm{~mm} \mathrm{mrad}\right.$, length $L_{b 0}=30 \mu \mathrm{m}(100 \mathrm{fs})$, and $\mathrm{rms}$ radius $R_{a \nu}^{0}=25-50 \mu \mathrm{m}$. Open squares: $\phi_{\max }=0.04, R_{a \nu}^{0}=50 \mu \mathrm{m}$; solid squares: $\phi_{\max }=0.19, \quad R_{a \nu}^{0}=50 \mu \mathrm{m} ; \quad$ solid circles: $\phi_{\max }=0.19$, $R_{a \nu}^{0}=25 \mu \mathrm{m}$. 
For smaller wakefield amplitude, the required compression distance increases and the final bunch radius also increases substantially, as demonstrated in Fig. 4(b).

More detailed analysis of bunch compression for the wakefield amplitude $\phi_{\max }=0.19$ depending on the initial bunch size $\left(R_{a \nu}^{0}=50-100 \mu \mathrm{m}, L_{b 0}=30-90 \mu \mathrm{m}\right)$ and emittance $\left(\varepsilon_{0}=0.3-0.6 \mathrm{~mm} \mathrm{mrad}\right)$ is presented in Fig. 5 . Simulations show the importance of providing a small ra-
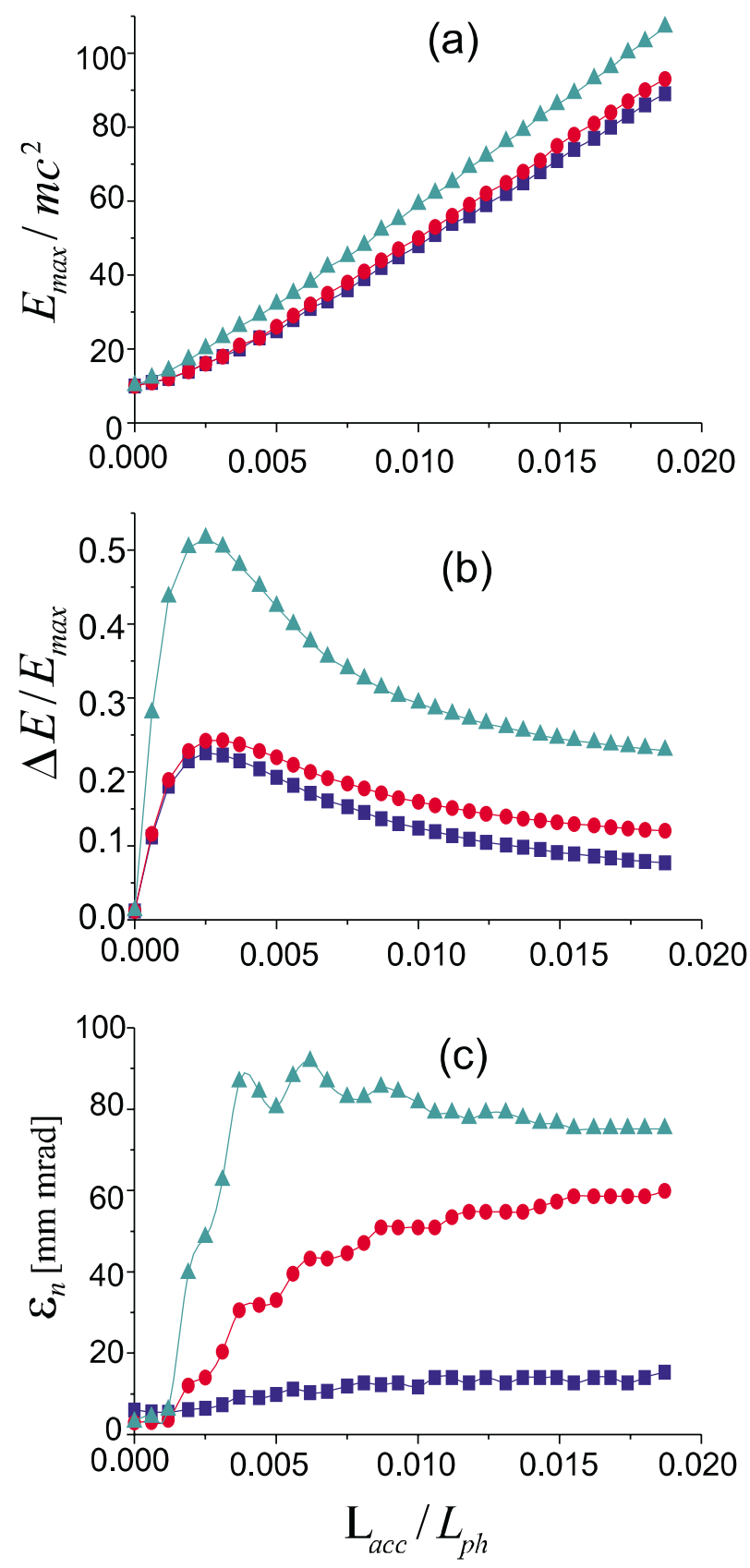

FIG. 5. (Color) The effect of the initial bunch size on the beam quality in the bunching stage; $\phi_{\max }=0.19$, other laser and plasma channel parameters are similar to Fig. 4. Triangles: $L_{b 0}=90 \mu \mathrm{m}(300 \mathrm{fs}), R_{a \nu}^{0}=100 \mu \mathrm{m}$, $\varepsilon_{0}=0.3 \mathrm{~mm}$ mrad; $\quad$ circles: $L_{b 0}=30 \mu \mathrm{m} \quad(100 \quad \mathrm{fs})$, $R_{a \nu}^{0}=100 \mu \mathrm{m}, \quad \varepsilon_{0}=0.3 \mathrm{~mm} \mathrm{mrad}$; squares: $L_{b 0}=30 \mu \mathrm{m}$ $(100 \mathrm{fs}), R_{a \nu}^{0}=50 \mu \mathrm{m}, \varepsilon_{0}=0.6 \mathrm{~mm}$ mrad. dius and duration of the electron bunch at the entrance to the bunching stage not only for effective bunch compression but for a low energy spread and emittance as well.

Note that deviation of the injection moment by $\delta t$ to compare with the optimum injection phase at the maximum of the potential results, according to Eq. (11), in extension of the compressed bunch length by $\delta L_{b} / L_{b}=$ $2 c \delta t / L_{b 0}$. This proportion indicates that the jitter between the electron and laser pulses needs to be small to compare with $L_{b 0}$.

\section{MONOENERGETIC LASER WAKEFIELD ACCELERATION OF COMPRESSED ELECTRON BUNCHES}

In view of the obtained (in Secs. II and III) dependencies of the LWFA performance upon the initial bunch parameters, the optimum LWFA scheme evolves into the two-stage design where the first stage serves for bunch compression and the second for monoenergetic acceleration. For the first bunching stage, we again consider the $5 \mathrm{MeV}$ injected electron bunch with parameters shown in the caption of Fig. 5. Laser and plasma parameters are taken similar for both stages and the same as in the simulations discussed in Sec. III.
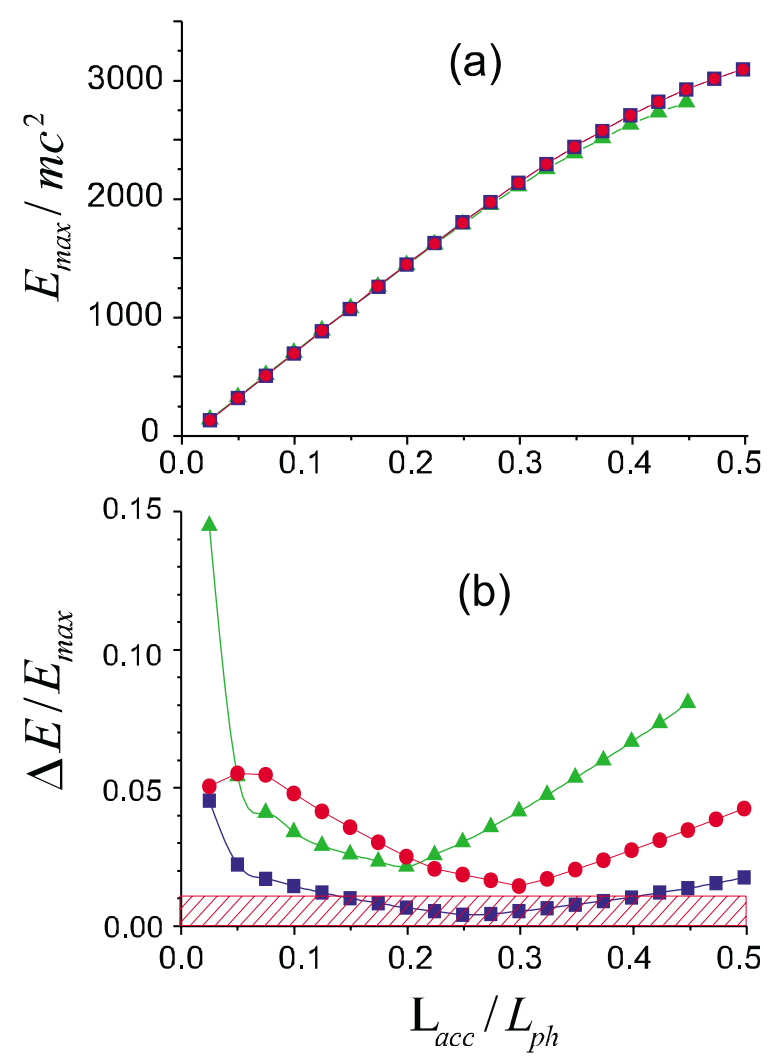

FIG. 6. (Color) Acceleration of electron bunches extracted from the bunching stage. All the parameters are similar to those given in the caption of Fig. 5. 

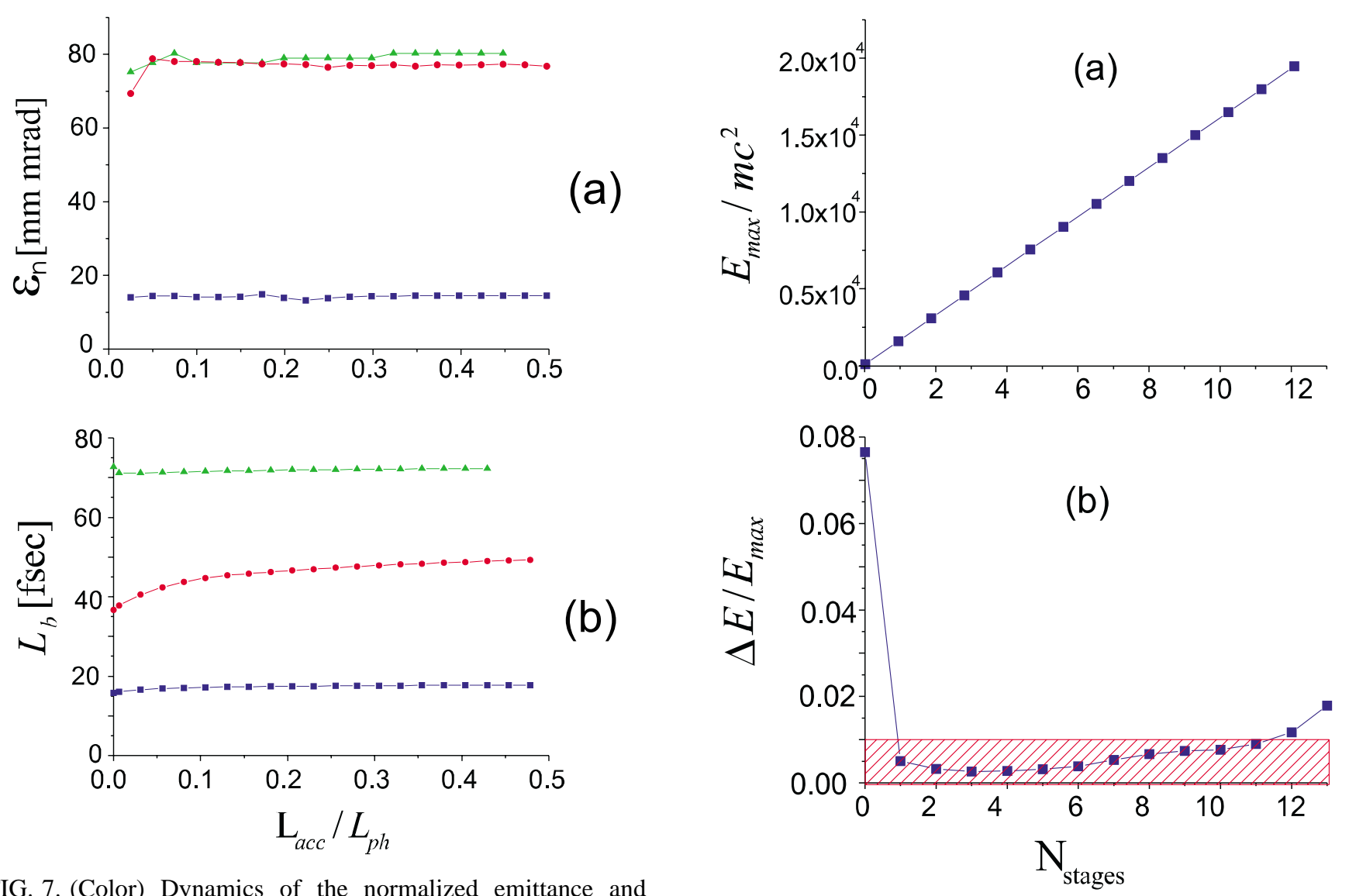

FIG. 7. (Color) Dynamics of the normalized emittance and bunch duration in the accelerator stage. All the parameters are similar to those given in the caption of Fig. 5.

The electron bunch with the initial parameters $R_{a \nu}^{0}=$ $50 \mu \mathrm{m}, L_{b 0}=30 \mu \mathrm{m}, \varepsilon_{0}=0.6 \mathrm{~mm} \mathrm{mrad}$ is extracted from the bunching stage at the resonant energy of $40 \mathrm{MeV}$ and compressed down to $20 \mathrm{fs}$. Then, this bunch is injected into the accelerating stage in the vicinity of the maximum accelerating field at the beginning of the focusing phase. Figure 6 shows that acceleration up to $1.5 \mathrm{GeV}$ with the energy spread of $<2 \%$ can be observed in the single LWFA stage extending over the whole focusing phase.

An important result is the preservation of the normalized emittance and bunch duration in the course of the acceleration (see Fig. 7). The observed preservation of the emittance and bunch length during the acceleration allows for the multistage LWFA. Figure 8 shows the energy gain and spread for a number of accelerating stages (for the same laser and plasma parameters as given previously).

\section{CONCLUSIONS}

In this paper, we demonstrate the possibility of generating quasimonoenergetic electron beams with a controlled emittance in the plasma-channel LWFA, providing that the injected electron bunch is a small fraction of the plasma wavelength both longitudinally and transversely.

FIG. 8. (Color) Multistage LWFA with prebuncher; initial electron bunch parameters (before bunching stage) are $L_{b 0}=$ $30 \mu \mathrm{m}(100 \mathrm{fs}), R_{a \nu}^{0}=50 \mu \mathrm{m}, \varepsilon_{0}=0.6 \mathrm{~mm} \mathrm{mrad}$.

Upon proper scaling, the obtained results are applicable to LWFA with any type of laser driver. For example, the concept of the two-stage LWFA with a prebuncher may be generally useful regardless of the wavelength of the driver laser.

The particular example of a $\mathrm{CO}_{2}$ laser driver demonstrates the feasibility of applying a conventional photocathode rf gun as an injector for the advanced LWFA that satisfies the goals for the next generation laser accelerator experiment. In addition, the long wavelength of the $\mathrm{CO}_{2}$ laser permits a favorable scaling of the plasma wavelength and channel that relaxes experimental tolerances to alignment and synchronization and allows a proportional increase of the accelerated bunch charge. Three dimensional particle-in-cell simulations are in progress to provide a quantitative estimate to the maximum bunch charge below the threshold of the wakefield degradation due to the spatial self-charge.

\section{ACKNOWLEDGMENTS}

This work was supported by Russian Foundation for Basic Research under Grant No. 98-02-16263 and by the U.S. DOE under Contract No. DE-AC02-76CH00016. 
[1] A. Madena, Z. Najmudin, A.E. Dangor, C.E. Clayton, K. A. Marsh, C. Joshi, V. Malka, C. B. Darrow, C. Danson, D. Neely, and F. N. Walsh, Nature (London) 377, 606 (1995).

[2] K. Nakajima, D. Fisher, T. Kawakubo, H. Nakanishi, A. Ogata, Y. Kato, Y. Kitagawa, R. Kodama, K. Mima, H. Shiraga, K. Suzuki, K. Yamakawa, T. Zhang, Y. Sakawa, T. Shoji, Y. Nishida, N. Yugami, M. Downer, and T. Tajima, Phys. Rev. Lett. 74, 4428 (1995).

[3] M. Everett, A. Lal, D. Gordon, C. Clayton, K. Marsh, and C. Joshi, Nature (London) 368, 527 (1994).

[4] D. Umstadter, S.-Y. Chen, A. Maksimchuk, G. Mourou, and R. Wagner, Science 273, 472 (1996).

[5] D. Umstadter, J. K. Kim, and E. Dodd, Phys. Rev. Lett. 76, 2073 (1996).

[6] E. Esarey, R. F. Hubbard, W. P. Leemans, A. Ting, and P. Sprangle, Phys. Rev. Lett. 79, 2682 (1997).

[7] I. V. Pogorelsky, I. Ben-Zvi, J. Skaritka, Z. Segalov, M. Babzien, K. Kusche, I. K. Meshkovsky, V. A. Lekomtsev, A. A. Dublov, Yu. A. Boloshin, and G. A. Baranov, in Advanced Accelerator Concepts: Seventh Workshop, edited by S. Chattopadhyay, J. McCullough, and P. Dahl, AIP Conf. Proc. No. 398 (AIP, New York, 1997), p. 937.

[8] I. V. Pogorelsky, I. Ben-Zvi, M. Babzien, K. Kusche, J. Skaritka, I. Meshkovsky, A. Dublov, V. Lekomtsev, I. Pavlishin, and A. Tsunemi, in Proceedings of LASERS'97, New Orleans, Louisiana, 1997 (STS Press, McLean, VA, 1998), p. 861.

[9] I. V. Pogorelsky, I. Ben-Zvi, M. Babzien, K. Kusche, J. Skaritka, I. K. Meshkovsky, A. A. Dublov, V. A. Lekomtsev, I. V. Pavlishin, Y.A. Boloshin, G. B. Deineko, and A. Tsunemi (to be published).

[10] T. Tajima and J. M. Dawson, Phys. Rev. Lett. 43, 267
(1979).

[11] L. M. Gorbunov and V. I. Kirsanov, Sov. Phys. JETP 66, 290 (1987); L. M. Gorbunov and V. I. Kirsanov, in Nonlinear Theory of Strong Electromagnetic Wave-Plasma Interactions, edited by O. N. Krokhin, Proceedings of the Lebedev Physics Institute No. 213 (Nova, Commack, NY, 1993), p. 3.

[12] E. Esarey, P. Sprangle, J. Krall, and A. Ting, IEEE Trans Plasma Sci. 24, 252 (1996).

[13] W. Leemans, C. W. Siders, E. Esarey, N. Andreev, G. Shvets, and W. B. Mori, IEEE Trans. Plasma Sci. 24, 331 (1996).

[14] N.E. Andreev, A. A. Frolov, S. V. Kuznetsov, E. V. Chizhonkov, and L. M. Gorbunov, in Proceedings of LASERS'97, New Orleans, Louisiana, 1997 (Ref. [8]), p. 875.

[15] N.E. Andreev, L. M. Gorbunov, and E. V. Chizonkov, Russ. J. Numer. Anal. Math. Modeling 13, 1 (1998).

[16] N.E. Andreev, E. V. Chizhonkov, A.A. Frolov, and L. M. Gorbunov, Nucl. Instrum. Methods Phys. Res., Sect. A 410, 469 (1998).

[17] E. Esarey, J. Krall, and P. Sprangle, Phys. Rev. Lett. 72, 2887 (1994).

[18] N.E. Andreev, L. M. Gorbunov, and V. I. Kirsanov, Phys. Plasmas 2, 2573 (1995).

[19] I. V. Pogorelsky, in Proceedings of LASERS'97, New Orleans, Louisiana, 1997 (Ref. [8]), p. 868.

[20] X. J. Wang, X. Qiu, and I. Ben-Zvi, Phys. Rev. E 54, R3121 (1996).

[21] N.E. Andreev, L.M. Gorbunov, V.I. Kirsanov, K. Nakajima, and A. Ogata, Phys. Plasmas 4, 1145 (1997).

[22] N. E. Andreev and S. V. Kuznetsov, Bull. Lebedev Phys. Inst. 1, 9 (1999). 